\title{
Oculocardiac reflex in an adult with a trapdoor orbital floor fracture: case report, literature review, and differential diagnosis
}

\author{
Bernardo Ferreira Brasileiro ${ }^{1}$, Joseph E. Van Sickels ${ }^{2}$, Larry L. Cunningham, Jr. ${ }^{2}$ \\ ${ }^{1}$ Kentucky Clinic, ${ }^{2}$ Division of Oral and Maxillofacial Surgery, College of Dentistry, University of Kentucky, Lexington, KY, USA
}

\begin{abstract}
J Korean Assoc Oral Maxillofac Surg 2020;46:428-434)
Orbital floor blowout fractures can result in a variety of signs and symptoms depending on the severity of the bone defect. Large defects often result in enophthalmos and restriction of ocular movement; yet the timing of surgery can be delayed up to two weeks with good functional outcomes. In contrast, an orbital trapdoor defect with entrapment of the inferior rectus muscle usually elicits pain with marked restriction of the upward gaze and activation of the oculocardiac reflex without significant dystopia or enophthalmos. When autonomic cardiac derangement is diagnosed along with an orbital floor fracture, it has been suggested that the fracture should be treated immediately. Otherwise, it will result in continued hemodynamic instability and muscular injury and may require a second surgery. This article reports the management of an unusual presentation of a trapdoor blowout orbital floor fracture surgery with oculocardiac response in an adult, with emphasis on its pathophysiology, management, and differential diagnosis.
\end{abstract}

Key words: Orbital fractures, Facial injuries, Oculocardiac reflex

[paper submitted 2018. 10. 9 / revised 2018. 10. 31 / accepted 2018. 11. 17]

\section{Introduction}

Orbital floor fractures can present in isolation or in combination with other facial fractures. The etiology is usually direct trauma to the orbital region resulting in fracture to the orbital floor'. The fracture pattern is usually an "open door" defect and is typically seen in adults with herniation of the orbital contents into the maxillary sinus. Clinically, there may be periorbital bruising, subconjunctival hemorrhage, diplopia, altered sensation of the infraorbital nerve, and enophthalmos. Any limitation in ocular movement is usually due to pain or "relative entrapment" of the ocular muscles ${ }^{2}$.

Greater elasticity of the facial skeleton in children can result in a slightly different fracture pattern, thus changing the clinical presentation ${ }^{3}$. Following orbital trauma in a child

\footnotetext{
Bernardo Ferreira Brasileiro

Kentucky Clinic, University of Kentucky, Adult Dentistry, 740 South Limestone, Lexington, KY 40508, USA

TEL: +1-859-323-8873 FAX: +1-859-257-3270

E-mail:bernardobrasileiro@uky.edu

ORCID: https://orcid.org/0000-0002-5886-267X

(c) This is an open-access article distributed under the terms of the Creative Commons Attribution Non-Commercial License (http://creativecommons.org/ licenses/by-nc/4.0/), which permits unrestricted non-commercial use, distribution, and reproduction in any medium, provided the original work is properly cited.

Copyright (C) 2020 The Korean Association of Oral and Maxillofacial Surgeons. All rights reserved.
}

who has softer, more elastic bones, an impact can result in a fracture that first hinges open, springing inferiorly, and then returns to the original position, thus entrapping tissue. Small floor fractures are more likely to entrap extraocular muscles, leading to a compartment syndrome, which if not promptly relieved can lead to prolonged and possibly permanent paresis and muscular scarring. The usual presentation of a linear fracture of the orbital floor or medial wall can occur as a "trapdoor" with incarceration of orbital soft tissue and muscles accompanied by little sign of soft tissue injury ${ }^{4}$. This type of fracture has been called the white-eyed orbital blowout fracture (WEOBF) and was first named in a report by Jordan et al. ${ }^{5}$. They described it occurring in a group of young patients (less than 16 years of age) with little or no clinical evidence of soft tissue trauma, diplopia with restriction of the vertical gaze, lack of exophthalmos, and radiographic signs of minimal bone displacement with or without tissue herniation into the maxillary sinus. In an even more serious scenario, these patients may have true entrapment of the inferior rectus, pain with restricted ocular movement, and autonomic signs, which are secondary to the oculocardiac reflex $(\mathrm{OR})^{6}$.

The OR is a vagally-mediated slowing of the heart following painful stimulation of the orbital contents through an arc reflex response, which can result in a $20 \%$ or more reduction in heart rate, the presence of arrhythmias, or both ${ }^{7,8}$. The first 
description of this reflex was simultaneously but independently reported in 1908 by Aschner ${ }^{9}$ and Dagnini ${ }^{10}$. They described a pressure-induced neural reflex causing cardiac depression through vagal stimulation, and the phenomenon was labeled "oculocardiac reflex". For centuries before their reports, pressure on the globes had been used therapeutically to treat paroxysmal atrial tachycardia ${ }^{11}$. Nowadays, the OR is a well-known condition in ophthalmology, anesthesiology, otolaryngology, neurology, and maxillofacial surgery. Typical presenting signs and symptoms include bradycardia, alterations in blood pressure, faintness, and nausea, when any of the peripheral branches or the central component of the trigeminal nerve are stimulated ${ }^{12}$. The OR has been documented during temporomandibular joint surgery and mandibular osteotomies, emphasizing that the maxillary and mandibular divisions of the trigeminal nerve can be involved as the ophthalmic branch ${ }^{4}$. In 1988, Shelly and Church ${ }^{13}$ introduced the term "trigeminocardiac reflex" to describe the overall mechanism, clarifying that the OR, although most commonly elicited, is a particular manifestation of a more general reflex phenomenon. Therefore, any surgical intervention in the distribution of the trigeminal nerve poses a risk of precipitating a cardiovascular incident in response to a trigeminal stimulation $^{14}$.

The WEOBF in a pediatric patient is an often cited cause for inducing the OR. In this report, the authors describe an unusual presentation of an orbital floor blowout fracture with entrapment of orbital contents, minimal soft tissue trauma signs, and elicited OR during orbital floor fracture surgery in an adult patient. The article also aims to discuss the pathophysiology of the OR and its management in orbital floor fractures with muscle entrapment, as well as reviewing a differential diagnosis in a trauma patient.

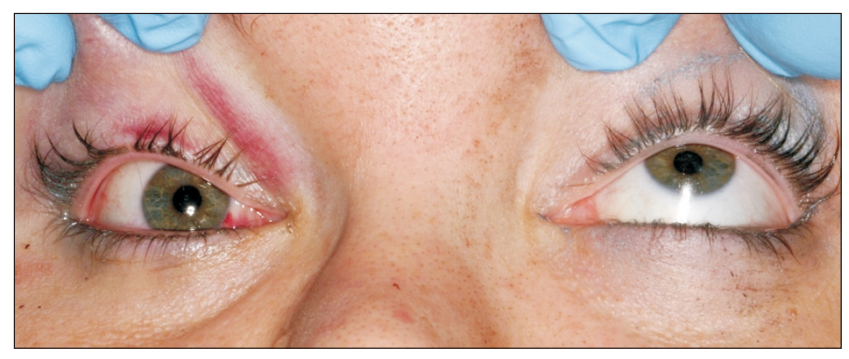

Fig. 1. Preoperative clinical examination of the upward gaze, demonstrating vertical restriction in ocular motility after an orbital floor blowout fracture on the right side.

Bernardo Ferreira Brasileiro et al: Oculocardiac reflex in an adult with a trapdoor orbital floor fracture: case report, literature review, and differential diagnosis. J Korean Assoc Oral Maxillofac Surg 2020

\section{Case Report}

A 26-year-old white female was seen following a motor vehicle collision. She was an unrestrained backseat passenger who hit her face against the front seat backrest. Her vital signs showed stable blood pressure readings, normal heart and respiratory rates, and regular temperature. However, she had nausea, vomiting, and syncope with attempts to move the right eye. Visual acuity was 20/20 OU. There was no loss of globe integrity, enophthalmos, or hypoglobus. No afferent pupillary defect or infraorbital nerve paraesthesia was detected. Minimal subconjunctival and upper eyelid ecchymosis was observed on the affected side. Remarkably, eye pain, diplopia, and dizziness were noted and increased with attempts at a superior gaze. The patient was found to have severe impairment of superior and limited inferior gaze of the right eye.(Fig. 1) Coronal computed tomography (CT) scans with $1-\mathrm{mm}$ sections revealed a small disruption in the median aspect of the inferior floor of the right orbit, which was compatible with entrapment of periorbital soft tissue contents.(Fig. 2) Cerebral and spinal injuries were ruled out after clinical and tomographic evaluations. There were no chest or abdominal injuries noted.

Based on the radiographic findings of orbital content entrapment and the clinical signs of impaired globe movement with uncontrolled nausea, a diagnosis of an orbital floor fracture with potential risk for an OR was established. A decision was made to take the patient immediately to the operating room to release the entrapped inferior rectus muscle and to reconstruct the orbital floor. Early intervention was deemed necessary to prevent muscle ischemia and to improve ocular motility. The risk of OR exacerbation was discussed with the anesthesiology team prior to surgery.

The patient underwent an uneventful induction and oral intubation in the operating room within 4 hours after pre-

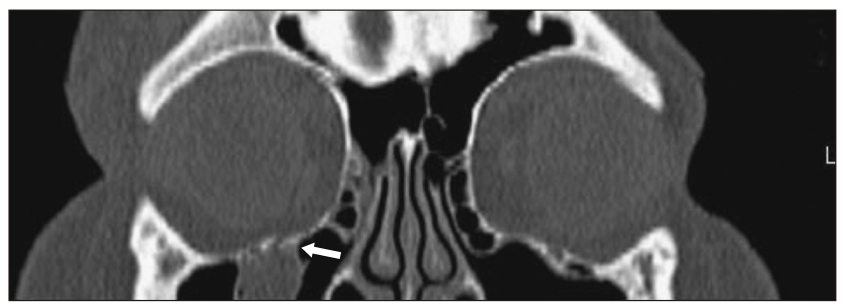

Fig. 2. Preoperative coronal computed tomography (coronal view) showing right floor linear discontinuity with entrapment of orbital contents (white arrow) into the maxillary sinus (tear drop sign).

Bernardo Ferreira Brasileiro et al: Oculocardiac reflex in an adult with a trapdoor orbital floor fracture: case report, literature review, and differential diagnosis. J Korean Assoc Oral Maxillofac Surg 2020 
sentation to the Emergency Department. Periorbital tissue infiltration with local anesthetics was administered. A forced duction test revealed marked restriction in both the superior and inferior movements of the eye. A transconjunctival incision was made, exposing the fracture. The inferior periorbital soft tissue was visualized trapped between fractured orbital floor segments. The inferior rectus muscle was also identified within the fracture line.(Fig. 3) During the dissection, severe short-term bradycardia (decrease in heart rate from 66 beats/ min to 25 beats/min) was noted, which immediately resolved with release of the muscle and completion of orbital content manipulation. Following muscle releasing, the fracture line of the floor showed no actual defect. Due to instability of the reduced bony segment, a resorbable orbital plate was placed without fixation (Synthes Rapid Resorbable Fixation System, West Chester, PA, USA).(Fig. 4) A forced duction test after implant placement showed normal globe motility. Immediately after surgery, visual acuity was intact, no oculocardiac symptom or nausea was reported, and the patient had normal ocular movements, as presented in Fig. 5. Hospital discharge occurred the following day, and the patient was followed up weekly. Thirty days after surgery, she had residual complaints of mild soreness in the extreme upward gaze without restriction. An ophthalmologist who saw the patient recommended no additional intervention. At 2 months after surgery, she had no further complaints or symptoms.

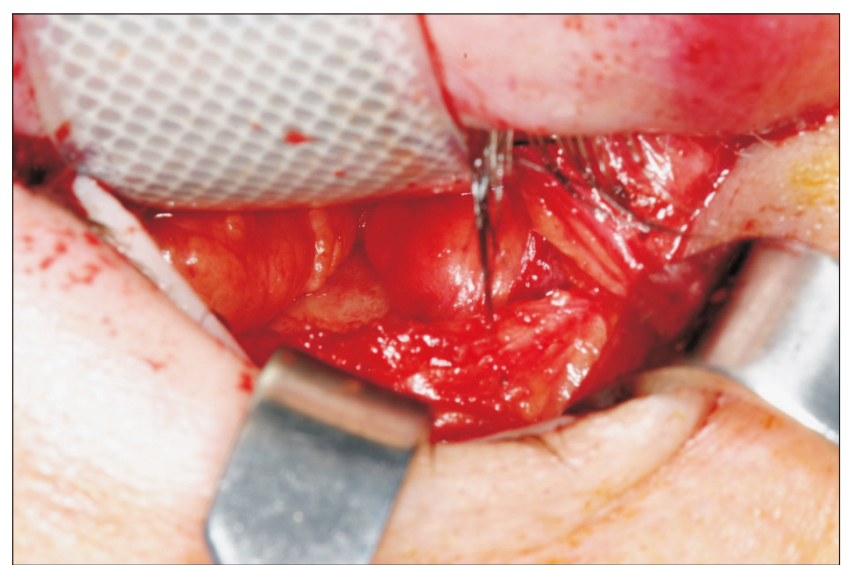

Fig. 3. Intraoperative view of the right orbit with linear floor blowout fracture and trapdoor defect. Entrapment of the inferior rectus muscle and orbital fat can be observed.

Bernardo Ferreira Brasileiro et al: Oculocardiac reflex in an adult with a trapdoor orbital floor fracture: case report, literature review, and differential diagnosis. J Korean Assoc Oral Maxillofac Surg 2020

\section{Discussion}

The linear defect in the trapdoor blowout fracture suggests that buckling of the thin orbital walls with subsequent entrapment of the adjacent soft tissues is the most likely mechanism responsible for this pattern of orbital trauma ${ }^{15}$. The greater elasticity of the bones in young patients explains the propensity for this fracture pattern in children, and the minimal bone damage develops little to no signs in the surrounding tissues. Although it has been suggested that WEOBF is observed almost exclusively in children, a few reports have recently highlighted that it can occur in adults ${ }^{1,3}$. As noted in this case report, we observed similar features of a trapdoor WEOBF in an adult, who presented minimal periorbital fracture signs and associated nausea because of extraocular muscle entrapment. Although classic blowout fractures are rarely associat-

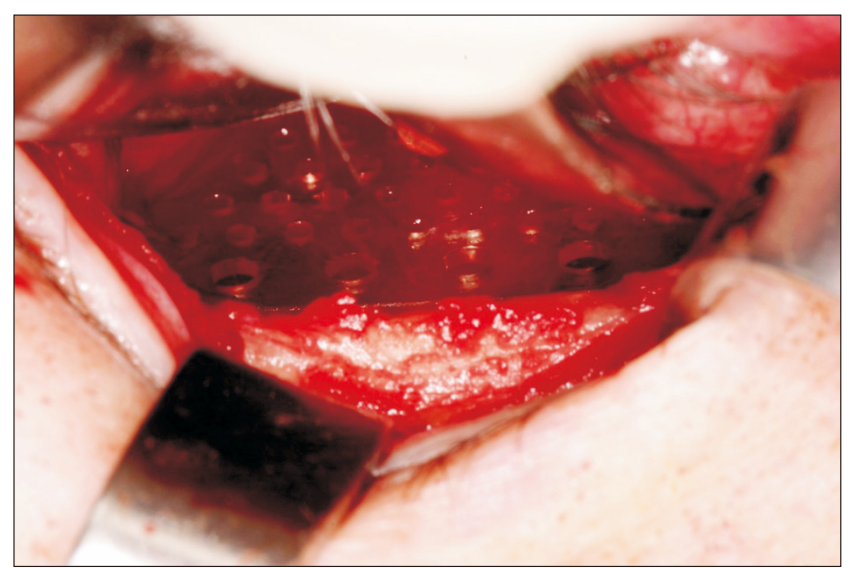

Fig. 4. Intraoperative view of orbital floor reconstruction with a resorbable orbital plate after incarcerated soft tissue release and floor fracture reduction.

Bernardo Ferreira Brasileiro et al: Oculocardiac reflex in an adult with a trapdoor orbital floor fracture: case report, literature review, and differential diagnosis. J Korean Assoc Oral Maxillofac Surg 2020

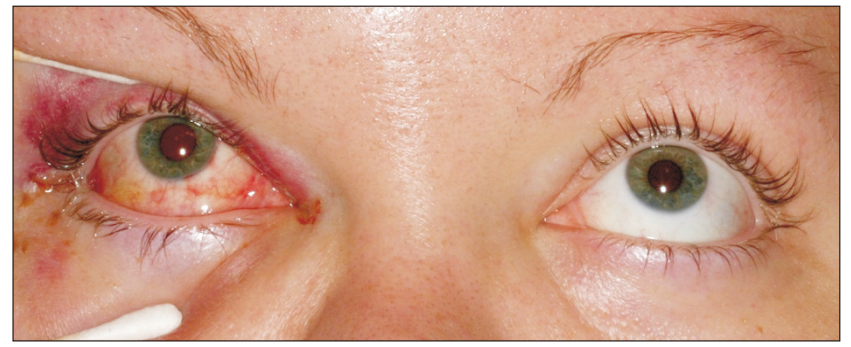

Fig. 5. Immediate postoperative clinical examination of the upward gaze, exhibiting normal vertical ocular motility after management of the orbital floor blowout fracture on the right side.

Bernardo Ferreira Brasileiro et al: Oculocardiac reflex in an adult with a trapdoor orbital floor fracture: case report, literature review, and differential diagnosis. J Korean Assoc Oral Maxillofac Surg 2020 
ed with serious autonomic symptoms, the presence of painful restriction in ocular motility associated with nausea or vomiting should alert the clinician to the possibility of an orbital "trapdoor" fracture with significant risk for the development of hemodynamic changes. Finally, the OR was elicited during direct manipulation of the periorbital tissue to release the entrapped soft tissue. This article reports on an unusual presentation of a trapdoor blowout orbital floor fracture surgery with oculocardiac response in an adult with emphasis on its pathophysiology, management, and differential diagnosis.

The OR occurs because of an arc reflex response between the eye and the heart, demonstrating a direct link between cranial nerves $\mathrm{V}$ and $\mathrm{X}$. The onset of the $\mathrm{OR}$ is precipitated by a course of afferent impulses along the ophthalmic branch of the trigeminal nerve via the ciliary nerves of the globe. Neuronal signals travel along the ciliary ganglion, nasociliary nerve, ophthalmic nerve, and trigeminal ganglion until they reach the sensory nucleus of the trigeminal nerve in the brainstem. Further from the trigeminal nerve nucleus, the afferent reflex arm is connected to the efferent pathway via the short internuncial fibers in the reticular formation, connecting the motor nucleus of the vagus nerve to the endings of the branches in the myocardium at the sinoatrial node.(Fig. 6) The vagus nerve is responsible for the efferent impulses of the OR, which increases parasympathetic tone to the heart, which may mediate negative cardiac chronotropic and inotropic responses, leading to bradycardia and hypotension. Vagal stimulation is also correlated with symptoms of nausea and vomiting due to gastric hypermobility ${ }^{4,14,15}$.

Clinically, the OR has been observed in procedures involving direct or indirect manipulation of the orbit or orbital contents, especially of the medial rectus and inferior oblique muscles ${ }^{6,8,11}$. In maxillofacial trauma, precipitation of an OR can include spontaneous eye movement during ophthalmologic assessment, intraoperative direct manipulation of orbital contents, or indirect stimulus to periorbital tissue after reduction of zygomaticomaxillary complex fractures. Although nausea/vomiting and cardiac symptoms appear to be predictive of a muscular incarceration in blowout fractures ${ }^{16}$, entrapment of the extraocular muscles is not needed to elicit the arc flex, since large orbital floor blowout fractures may result in enough tension on the inferior rectus muscle as to induce the $\mathrm{OR}^{6}$. Fahling and McKenzie ${ }^{17}$ reported a case of a 26-year-old white man who sustained a self-inflicted gunshot wound under his right mandible who developed progressive proptosis and a decrease in heart rate to 43 beats $/ \mathrm{min}$. The authors considered that stretch of the extraocular musculature and increased intraorbital pressure caused by a retrobulbar hematoma to have initiated the afferent limb of the OR, resulting in bradycardia. Emergent lateral canthotomy and cantholysis were performed to offset the negative chronotropic effect of the OR. After the procedure, the patient's heart rate stabilized in the $60 \mathrm{~s}$, and no further episodes of bradycardia were noted during the subsequent few hours he remained in

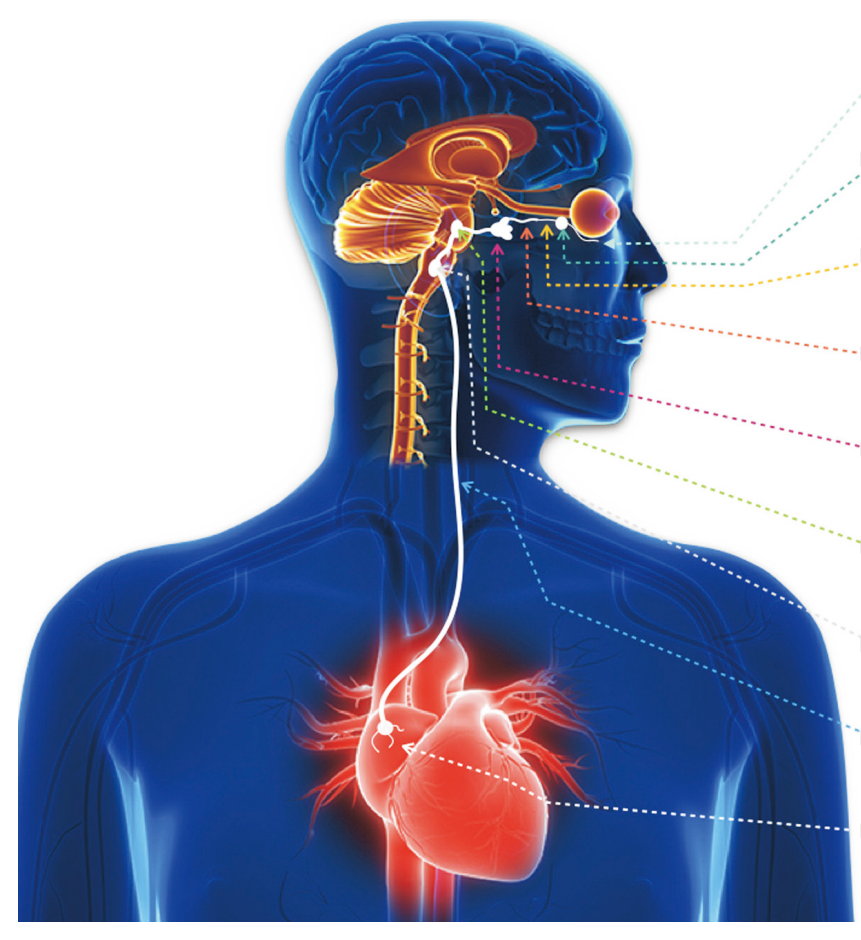

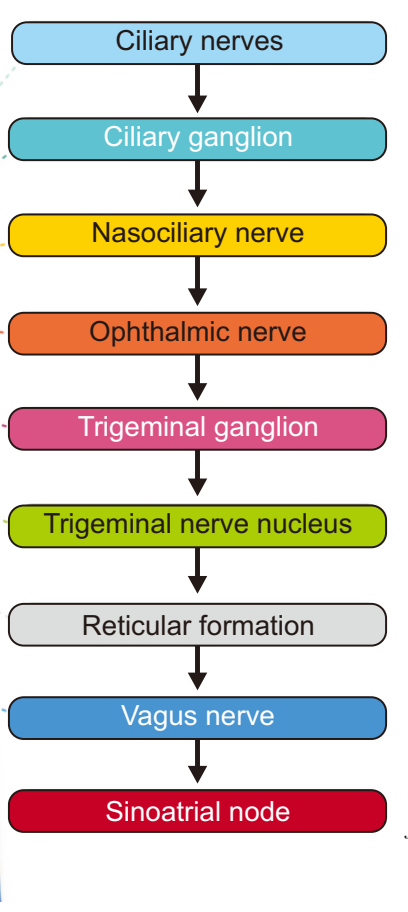

Fig. 6. Graphic illustration of the oculocardiac reflex pathway from afferent sensory nerve endings of the trigeminal nerve (ciliary nerves) to efferent branches of the vagus nerve in the heart (sinoatrial node).

Bernardo Ferreira Brasileiro et al: Oculocardiac reflex in an adult with a trapdoor orbital floor fracture: case report, literature review, and differential diagnosis. J Korean Assoc Oral Maxillofac Surg 2020 
the Emergency Department while awaiting disposition from the ophthalmology and trauma services.

The result of OR onset may generate a series of cardiac abnormalities, which may include slowness of sinus rhythm, depression of intracardiac conduction, and depression of myocardial contractility, with an incidence rate of 1 in 3,500 cases $^{8}$. The OR may result in autonomic instability, including episodes of bradycardia below 25 beats/min, brief periods of asystole, and even death if left untreated ${ }^{4,7,11,12}$. According to $\mathrm{Smith}^{18}$, there are at least 3 reports of death that can be attributed to the OR in the literature. In 1956, Sorenson and Gilmore $^{19}$ reported a cardiac arrest during strabismus surgery. One year later, Kirsch et al. ${ }^{20}$ reported the death of a patient during surgery for retinal detachment in whom fatal cardiac arrest developed shortly after the preliminary manipulation of two extraocular muscles. Another death was reported by Bi$\mathrm{etti}^{21}$ in 1966, who reported a case of irreversible cardiac arrest during general anesthesia in a boy of eight years operated on for recession of the medial rectus. Thus, it is of paramount significance that the correct etiology of the cardiac arrhythmias and hemodynamic changes in patients sustaining orbital trauma or undergoing ophthalmologic/orbital surgery be established in order to institute prompt measures to achieve cessation of the OR.

In an emergency trauma setting, a comprehensive differential diagnosis for any of the autonomic symptoms observed in the OR should be addressed. For hypotension, it may include systemic conditions such as hemorrhage, cardiac tamponade, pneumo- or hemothorax, cardiogenic or neurogenic shock, pharmacologic or toxicologic agents, and fat or air embolism. Meanwhile, factors that may cause bradycardia in this setting include hypothermia, prolonged hypoxemia, pressure on the carotid sinus, or pharmacologic agents ${ }^{12}$. Since dizziness, altered vision, nausea, and vomiting are classic symptoms presented in head and neck trauma, the emergency physician must provide expeditious neurologic physical and tomographic evaluation to discard traumatic brain injuries. In these cases, wide-ranging physical and psychological effects due to direct damage to the brain tissue, which can cause severe long-term complications and death, should be included in the differential diagnosis ${ }^{11}$. In the present case, the patient had sustained mild facial trauma, and her initial physical and radiographic assessments were negative for intracranial abnormalities, spinal fractures, long bone fractures, or significant intra-abdominal injuries. Furthermore, the diagnosis of OR was supported by nausea induced during orbital examination, limited upward gaze in the affected eye, and CT imaging showing inferior rectus muscle entrapment. Autonomic signs of cardiac dysfunction were confirmed by elicitation of the OR during intraoperative manipulation of the orbital content (muscle releasing).

Predisposing and risk factors for the OR are children (increased vagal tone in younger patients), males, hypercarbia, hypoxemia, light anesthesia, $\beta$-adrenergic blockers, neuromuscular blocking agents, and potent narcotics (which may augment vagal tone by inhibiting the sympathetic nervous system $)^{12}$. The duration of the stimulus and the strength applied on the ocular musculature also exert significant influence on triggering the OR. A period of stimulation of 15 to 20 seconds is necessary to precipitate the OR, and its autonomic consequences are directly proportional to the amount of tension and the progression of the stimulus (manipulation) being applied to the extraocular muscles ${ }^{8}$. Besides, Lübbers et al. ${ }^{4}$ highlighted the necessity for precaution when handling at-risk patients (i.e., patients with a medical history of cardiac disease) and high-risk surgeries (e.g., for strabismus). Another trigger point listed by those authors is rinsing with cold water $\left(0^{\circ} \mathrm{C}\right.$ to $\left.20^{\circ} \mathrm{C}\right)$ at the distribution of the ophthalmic division of the trigeminal nerve. Alternatively, very deep anesthesia and retrobulbar block were found to decrease risk via blockage of the afferent limb ${ }^{12}$.

The oral and maxillofacial surgeon should remember that the OR could occur at any time there is manipulation around the globe. By identifying at-risk patients or procedures and by monitoring the electrocardiogram for arrhythmias and bradycardia, early detection is possible and, fortunately, the heart rebounds dramatically on termination of the stimulus ${ }^{6}$. In orbital surgery, the nature of the stimulus is the most important risk factor in inducing this reflex; therefore, abrupt and sustained manipulation of the trigeminal nerve should be avoided. Persistent cases respond to anticholinergic drugs, adrenaline, or associated infiltration of local anesthetics along the trigeminal nerve branch $^{12}$. Intravenous atropine $(0.5 \mathrm{mg}$ increments) or glycopyrrolate are the drugs of choice in cases of refractory bradycardia or asystole ${ }^{11}$. Prophylactic preoperative administration of vagolytic agents in orbital fracture surgery and elective maxillofacial procedures has not been well established. It has been found to be ineffective in longer surgical procedures, in patients presenting with tachycardia from a hypovolemic event, and due to the other risks of cardiac dysrhythmias associated with the use of atropine ${ }^{15}$.

The management of blowout fractures has remained debatable, particularly with regard to the indications for and the timing of treatment ${ }^{3}$. Management strategies regarding 
treatment of orbital fractures vary regarding indications for surgery, timing of intervention, and practice patterns within and across specialties. Definitions of the surgical indications for the repair of orbital floor fractures were described by Burnstine $^{2}$ after a meta-analysis of the literature over a 20year period. Orbital floor fractures showing orbital soft tissue entrapment associated with signs of the OR warrant immediate intervention (within 24 hours). The presence of symptoms such as diplopia with the positive forced duction test and CT evidence of orbital tissue entrapment or orbital floor fracture of less than $50 \%$ of the floor area, causing latent enophthalmos, were indications for surgery within 2 weeks. Later surgical interventions can be pursued for patients without muscular entrapment or visual deficits, especially because many surgeons may prefer to perform orbital surgery after the swelling has subsided but before fibrous adhesion development.

The consensus for the appropriate timing for intervention in WEOBF with orbital soft tissue entrapment and associated symptoms of OR is immediate surgical repair. The rationale of this approach is based on the patient's significant autonomic features and $\mathrm{CT}$ confirmation of a trapdoor fracture with soft tissue entrapment ${ }^{1,3,5,16,17}$. Traction of the extraocular muscles, compression of the eyeball, or spontaneous ocular movement can lead to an OR with a sudden onset of parasympathetic hypotension, apnea, or gastric hypermobility during stimulation of any of the sensory branches of the trigeminal nerve. In the present case, the OR was first elicited during physical examination of the patient (eye movement in an upward gaze). Patients with these characteristics should be considered for immediate (within 12 hours) surgical intervention in light of their unpleasant symptoms and the potential risk of fatal cardiac arrhythmias ${ }^{2,15}$. Immediate intervention is also recommended to prevent muscle ischemia, fibrosis, and persistent restricted motility secondary to muscle contu$\operatorname{sion}^{2,5,16}$. In the present case, intervention occurred within 4 hours of admission to the emergency department, and no sign of either ischemic necrosis of the inferior rectus muscle or restriction of eye movement immediately after surgery were noted. A resorbable alloplastic plate was used, which has been gaining popularity for the reconstruction of inferior orbital wall defects because of its ease of use for small defects, elimination of donor-site morbidity, reduced surgical timing, and non-skeletal growth restriction in pediatric patients ${ }^{22}$.

In summary, the WEOBF is a special type of orbital injury, and its clinical diagnosis can be challenging. Despite the relative paucity of clinical ocular signs in WEOBF, a thorough clinical evaluation of ocular motility and acuity, assessment of autonomic symptoms, and orbital CT imaging may prevent a delayed diagnosis and optimize management of the patient. In an emergency setting, the clinician must be aware of the potential for a WEOBF to be associated with OR and promptly provide adequate surgical intervention, as well as prevent clinically significant cardiac decompensation.

\section{ORCID}

Bernardo Ferreira Brasileiro, https://orcid.org/0000-00025886-267X

Joseph E. Van Sickels, https://orcid.org/0000-0001-65721739

Larry L. Cunningham, Jr., https://orcid.org/0000-00021174-7105

\section{Authors' Contributions}

B.F.B. was responsible for literature review, writing the manuscript draft, and designing images. J.E.V.S. and L.L.C., Jr. participated in the patient surgical management and images selection, critical appraisal and final revision of the manuscript. All authors read and approved the final manuscript version.

\section{Consent for Publishing Photographs}

Written informed consent was obtained from the patient for publication of this article and accompanying images.

\section{Conflict of Interest}

No potential conflict of interest relevant to this article was reported.

\section{References}

1. Mehanna P, Mehanna D, Cronin A. White-eyed blowout fracture: another look. Emerg Med Australas 2009;21:229-32. https://doi. org/10.1111/j.1742-6723.2009.01186.x

2. Burnstine MA. Clinical recommendations for repair of isolated orbital floor fractures: an evidence-based analysis. Ophthalmology 2002;109:1207-10; discussion 1210-1; quiz 1212-3. https://doi. org/10.1016/s0161-6420(02)01057-6

3. Ethunandan M, Evans BT. Linear trapdoor or "white-eye" blowout fracture of the orbit: not restricted to children. Br J Oral Maxillofac Surg 2011;49:142-7. https://doi.org/10.1016/j.bjoms.2010.03.012

4. Lübbers HT, Zweifel D, Grätz KW, Kruse A. Classification of potential risk factors for trigeminocardiac reflex in craniomaxillo- 
facial surgery. J Oral Maxillofac Surg 2010;68:1317-21. https://doi. org/10.1016/j.joms.2009.12.039

5. Jordan DR, Allen LH, White J, Harvey J, Pashby R, Esmaeli B. Intervention within days for some orbital floor fractures: the whiteeyed blowout. Ophthalmic Plast Reconstr Surg 1998;14:379-90. https://doi.org/10.1097/00002341-199811000-00001

6. Woernley TC, Wright TL, Lam DN, Jundt JS. Oculocardiac reflex in an orbital fracture without entrapment. J Oral Maxillofac Surg 2017;75:1716-21. https://doi.org/10.1016/j.joms.2017.03.014

7. Pandya VB, Conway RM, Conway R. A heart-stopping orbital injury. Med J Aust 2008;189:410.

8. Ziccardi VB, Russavage J, Sotereanos GC, Patterson GT. Oculocardiac reflex: pathophysiology and case report. Oral Surg Oral Med Oral Pathol 1991;71:137-8. https://doi.org/10.1016/00304220(91)90453-j

9. Aschner B. [Ueber einen bisher noch nicht heschriebenen reflex vom auge auf kreislauf und atmung: verschwinden des radialispulses bei druck auf das auge]. Wien Klin Wochenscr 1908;21:152930. German.

10. Dagnini G. [lntorno ad un riglesso provocato in alcune emi: legici co110 stimolo della corna e colla pressione sul bulbo oculare]. Bull Sci Med 1908;8:380-1. Italian.

11. Chesley LD, Shapiro RD. Oculocardiac reflex during treatment of an orbital blowout fracture. J Oral Maxillofac Surg 1989;47:522-3. https://doi.org/10.1016/0278-2391(89)90290-5

12. Pham CM, Couch SM. Oculocardiac reflex elicited by orbital floor fracture and inferior globe displacement. Am J Ophthalmol Case Rep 2017;6:4-6. https://doi.org/10.1016/j.ajoc.2017.01.004

13. Shelly MP, Church JJ. Bradycardia and facial surgery. Anaesthesia 1988;43:422. https://doi.org/10.1111/j.1365-2044.1988.tb09042.x

14. Bhargava D, Thomas S, Chakravorty N, Dutt A. Trigeminocardiac reflex: a reappraisal with relevance to maxillofacial surgery. J Maxillofac Oral Surg 2014;13:373-7. https://doi.org/10.1007/s12663013-0541-4
15. Kim BB, Qaqish C, Frangos J, Caccamese JF Jr. Oculocardiac reflex induced by an orbital floor fracture: report of a case and review of the literature. J Oral Maxillofac Surg 2012;70:2614-9. https://doi.org/10.1016/j.joms.2012.06.189

16. Cohen SM, Garrett CG. Pediatric orbital floor fractures: nausea/ vomiting as signs of entrapment. Otolaryngol Head Neck Surg 2003;129:43-7. https://doi.org/10.1016/s0194-5998(03)00487-x

17. Fahling JM, McKenzie LK. Oculocardiac reflex as a result of intraorbital trauma. J Emerg Med 2017;52:557-8. https://doi. org/10.1016/j.jemermed.2016.08.022

18. Smith RB. Death and the oculocardiac reflex. Can J Anaesth 1994;41:760. https://doi.org/10.1007/BF03015643

19. Sorenson EJ, Gilmore JE. Cardiac arrest during strabismus surgery; a preliminary report. Am J Ophthalmol 1956;41:748-52. https://doi. org/10.1016/0002-9394(56)91767-6

20. Kirsch RE, Samet P, Kugel V, Axelrod S. Electrocardiographic changes during ocular surgery and their prevention by retrobulbar injection. AMA Arch Ophthalmol 1957;58:348-56. https://doi. org/10.1001/archopht.1957.00940010360005

21. Bietti OB. Problems of anesthesia in strabismus surgery. Int Ophthalmol Clin 1966;6:727-37.

22. Chen CT, Chen YR. Update on orbital reconstruction. Curr Opin Otolaryngol Head Neck Surg 2010;18:311-6. https://doi. org/10.1097/MOO.0b013e32833aafd2

How to cite this article: Brasileiro BF, Van Sickels JE, Cunningham LL Jr. Oculocardiac reflex in an adult with a trapdoor orbital floor fracture: case report, literature review, and differential diagnosis. J Korean Assoc Oral Maxillofac Surg 2020;46:428-434. https://doi.org/10.5125/jkaoms.2020.46.6.428 